\title{
Ultrasound scanning in the detection of hepatic fibrosis and steatosis
}

\author{
S H SAVERYMUTTU, A E A JOSEPH, J D MAXWELL
}

\begin{abstract}
Hepatic steatosis and fibrosis produce abnormal echo patterns on ultrasound scanning, but the potential of ultrasound scanning for diagnosing these conditions in routine clinical practice is uncertain. A prospective comparative study of 85 patients with histologically assessed liver conditions was performed, and specificity was assessed in 76 patients with functional bowel disease who were presumed to have normal livers.

Histological examination showed steatosis ranging from mild to severe in $\mathbf{4 8}$ patients and fibrosis ranging from increased fibrous tissue to established cirrhosis in $\mathbf{3 5}$ patients. Ultrasound scanning accurately identified steatosis, recognising 45 cases (sensitivity $94 \%$ ) with a specificity of $84 \%$. Fibrosis was less reliably detected (sensitivity $57 \%$ and specificity $88 \%$ ). Of the 50 patients with alcoholic liver disease, $47(94 \%)$ yielded abnormal results on scanning. In the 76 patients with functional bowel disease there was only one false positive result, giving a specificity of $99 \%$ in this group.

As hepatic steatosis is the earliest change in alcoholic liver disease and seems to be of prognostic importance for the development of cirrhosis, ultrasound scanning provides an effective screening procedure, particularly in the occult alcoholic, who often presents with non-specific gastrointestinal complaints.
\end{abstract}

\section{Introduction}

Real time ultrasound scanning is accepted as the first line imaging investigation in patients with suspected liver disorders. Although the liver may be affected by a wide variety of diseases, two major patterns are seen: focal and diffuse disease. In focal disease-for

\footnotetext{
Departments of Medicine and Radiology, Ultrasound, and Nuclear Medicine, St George's Hospital Medical School, London SW17 0RE

$S$ H SAVERYMUTTU, MRCP, lecturer in medicine

A E A JOSEPH, FRCR, consultant radiologist

J D MAXWELL, FRCP, consultant physician and senior lecturer

Correspondence to: Dr Saverymuttu.
}

example, extrahepatic biliary obstruction or metastatic diseasemany series have confirmed the accuracy of ultrasound scanning in diagnosis, and this is valuable in clinical management. ${ }^{1-4}$ In parenchymal liver disease, however, despite the reported association of recognisably abnormal echo patterns with both steatosis and fibrosis, ${ }^{5}$ the value of scanning in diagnosis and management is uncertain. ${ }^{4}$ The major factor that has inhibited decision making based on ultrasound findings in patients with parenchymal liver disease is the doubt about the accuracy of scanning when performed in the routine clinical setting. Furthermore, the reported accuracy in selected groups of patients in both retrospective and prospective studies has varied greatly, particularly for patients with fatty infiltration, with sensitivities ranging from $9 \%$ in patients with alcoholic liver disease ${ }^{6}$ to $80 \%$ in patients with unselected liver disease.

We attempted to define the clinical value of routine ultrasound examination in patients with parenchymal liver disease by prospectively comparing the result of scanning with histological diagnosis based on liver biopsy specimens.

\section{Patients and methods}

In a prospective study over two years abdominal ultrasound examination was performed on 450 patients referred from a general gastroenterology or liver clinic because of upper abdominal discomfort, hepatomegaly, abnormal results of liver function tests, or suspected alcoholic liver disease.

Evaluation of the accuracy of ultrasound scanning in identifying liver disease was restricted to the 85 patients for whom results of histological examination of the liver were available within 28 days of the ultrasound scan, either from percutaneous biopsy or postmortem examination. Table I gives the full list of diagnoses for these 85 patients. Most were found to have alcoholic liver disease, but no advice about reducing intake of alcohol was given until after biopsy. Biopsy specimens showing fatty infiltration were graded according to the degree of steatosis as mild, moderate, or severe; and those showing fibrosis were classified according to whether or not cirrhosis was present (table II)

Comparison of results obtained by ultrasound and histological examination in a normal control group was not possible as biopsy is not justified in patients without evidence of liver disease. We therefore attempted to evaluate the specificity of ultrasound scanning by analysing its accuracy in all patients with a final diagnosis of irritable bowel disease who fulfilled the following criteria: normal results of routine liver function tests, normal haemoglobin and mean cell volume, admitted daily alcohol consumption of 
$<40 \mathrm{~g}$ in men and $<20 \mathrm{~g}$ in women, and body weight within $20 \%$ of ideal. Of the 450 patients entered into the prospective study, 76 fulfilled these criteria and were presumed to have normal livers.

Ultrasound scanning was performed by a single operator with a Technicare Autosector (real time) using a $5 \mathrm{mHz}$ transducer. The time gain compensation settings were identical for all patients when depth of beam penetration was evaluated. The overall gain and initial gain settings were kept within a narrow range. Time gain compensation settings were altered only after the presence of fat or fibrosis had been evaluated and if the deeper parts of the liver could not be evaluated adequately. When deeper beam penetration was needed to complete the examination a $3.5 \mathrm{mHz}$ transduce was used. The mean duration of each examination was 15 minutes, which included assessment of gall bladder, liver, and pancreas specifically and the rest of the abdomen. The operator, who was unaware of the patients' clinical details or laboratory findings, was asked to complete a standard protoco sheet, indicating, firstly, whether the liver was abnormal or normal and, secondly, whether the abnormality was indicative of the presence of steatosis or fibrosis, or both.

The criterion for steatosis was hyperechogenic liver tissue with fine, tightly packed echoes. With the $5 \mathrm{mHz}$ transducer posterior beam penetration was an inevitable finding. The degree of steatosis was assessed by the fall in echo amplitude with depth (rate of posterior beam attenuation), increasing discrepancy of echo amplitude between liver and kidney, and loss of echoes from the walls of the portal veins.

Mild steatosis was thus recognised by a slight increase in liver echogenicity, a slight exaggeration of liver and kidney echo discrepancy, and relative preservation of echoes from the walls of the portal vein. Moderate steatosis was accompanied by loss of echoes from the walls of the portal veins, particularly from the peripheral branches, resulting in a featureless appearance of the liver. In addition, greater posterior beam attenuation was found and a greater discrepancy between hepatic and renal echoes. Severe steatosis was recognised by a greater reduction in beam penetration, loss of echoes from most of the portal vein wall, including the main branches, and a large discrepancy between hepatic and renal echoes.

Fibrosis, when present without noticeable steatosis, was recognised by a coarse echo pattern. In contrast to steatosis, in patients with fibrosis the portal vein walls are preserved and may in fact be exaggerated and posterior beam attenuation is not a noticeable feature. Cirrhosis was diagnosed only in the presence of regeneration nodules and was best seen on the surface of the liver, causing an irregular outline. Recognising fibrosis in the presence of steatosis was the greatest problem, and in patients with both conditions fibrosis was reported only when coarse echoes ("pin head" echoes) developed within the fine echo pattern of steatosis.

\section{Results}

FOCAL LIVER DISEASE

Eight patients had focal liver disease (table I) (in three cases associated with an underlying cirrhosis), which was correctly diagnosed by ultrasound scanning in all eight.

\section{PARENCHYMAL LIVER DISEASE}

Overall assessment-Of the 67 patients with histological evidence of either steatosis or fibrosis, 59 yielded abnormal results on ultrasound scanning of the liver, resulting in a sensitivity of $87 \%$. An abnormal ultrasound finding was highly important, only two patients without histological evidence being incorrectly identified as having liver disease (specificity $89 \%$ ). One patient had increased lipofuscin on histological examination but no other abnormality. Overall, 47 of the 50 patients with alcoholic liver disease yielded abnormal ultrasound findings (sensitivity $94 \%$ ).

Steatosis-Ultrasound scanning correctly identified 45 of the 48 patients with steatosis (sensitivity 94\%). All patients with moderate and severe steatosis were correctly identified, while the three false negative results (table II) occurred in patients who had mild fatty infiltration on biopsy. Six patients yielded false positive results for steatosis (specificity $84 \%$ ), five of whom had established cirrhosis and one normal liver architecture but increased lipofuscin pigment. Of the patients with steatosis, $40(84 \%)$ had alcoholic liver disease. The other causes of steatosis were diabetes mellitus, drugs (amiodarone, methotrexate), congestive cardiac failure, and reactive hepatitis (four cases). The sensitivity of scanning compared with liver function tests as a marker of steatosis was difficult to assess as abnormal results of liver function tests were the major indication for biopsy. Two patients, however, with normal results of liver function tests and ultrasonic evidence of steatosis were found to have fatty infiltration and other signs of alcoholic disease on biopsy.
Fibrosis-Ultrasound scanning was less accurate in detecting liver fibrosis, correctly identifying 20 patients (sensitivity $57 \%$ ). Of the 15 patients with fibrosis missed on scanning, nine had concomitant steatosis that was correctly identified. Six false positive scans for fibrosis occurred (specificity $88 \%$ ); five of the patients had steatosis.

Ultrasound scanning was more accurate in the subgroup with cirrhosis, correctly identifying increased fibrosis in 15 out of 21 patients (sensitivity $71 \%$ ). Regeneration nodules, however, were found in only six of the 21 patients. The six patients with cirrhosis who yielded false negative results were quite heterogeneous. Two patients with primary biliary cirrhosis were thought to have normal livers on scanning, one patient with Wilson's disease and one with cryptogenic cirrhosis were incorrectly thought to have steatosis, and in two other patients with cirrhosis (one alcoholic, the other induced by methotrexate) steatosis was correctly diagnosed but cirrhosis missed.

\section{NORMAL LIVER}

Only one of the 76 patients with irritable bowel disease with presumed normal livers had an abnormal ultrasound pattern. This man admitted drinking 12 pints of beer at weekends (mean alcohol consumption $34 \mathrm{~g}$ daily) but yielded normal results to laboratory tests. Assuming that all 76 patients had normal liver histology, the specificity of scanning for liver disease in this group was $99 \%$.

TABLE I-Final clinical diagnosis for all patients

\begin{tabular}{|c|c|c|c|}
\hline & No of patients & & No of patients \\
\hline Alcoholic liver disease & 50 & Cirrhosis associated with & \\
\hline Cirrhosis & 13 & methotrexate & 1 \\
\hline Precirrhosis & 37 & Cirrhosis associated & \\
\hline Metastases ${ }^{\star}$ & 5 & with amiodarone & 1 \\
\hline Chronic active hepatitis & 4 & Steatosis associated with & \\
\hline Reactive hepatitis & 8 & diabetes mellitus & 1 \\
\hline Hepatocellular carcinoma & 3 & Epstein-Barr virus hepatitis & 1 \\
\hline Primary biliary cirrhosis & 3 & $\begin{array}{l}\text { Polyarteritis } \\
\text { Pas }\end{array}$ & 1 \\
\hline Normal & 4 & Wilson's disease & 1 \\
\hline Chronic persistent & & Congestive cardiac failure & $i$ \\
\hline hepatitis & 2 & Functional bowel disease & \\
\hline Cryptogenic cirrhosis & 2 & (biopsy not done) & 76 \\
\hline
\end{tabular}

Includes one patient with cryptogenic cirrhosis.

fIncludes two patients with alcoholic liver disease.

TABLE II-Histological findings and accuracy of ultrasound in detecting hepatic steatosis and fibrosis in 85 patients who underwent liver biopsy

\begin{tabular}{|c|c|c|c|c|c|}
\hline & \multirow[b]{2}{*}{ Histology } & \multicolumn{4}{|c|}{ Ultrasound } \\
\hline & & $\begin{array}{c}\text { False } \\
\text { negative }\end{array}$ & $\begin{array}{c}\text { Sensitivity } \\
(\%)\end{array}$ & $\begin{array}{c}\text { False } \\
\text { positive }\end{array}$ & $\begin{array}{c}\text { Specificity } \\
(\%)\end{array}$ \\
\hline Total steatosis & 48 & 3 & 94 & & \\
\hline Severe & 11 & & 100 & & \\
\hline Moderate & 15 & & 100 & & \\
\hline Mild & 22 & 3 & 86 & & \\
\hline No steatosis & 37 & & & 6 & 84 \\
\hline Total fibrosis & 35 & 15 & 57 & & \\
\hline Cirrhosis & 21 & 6 & 71 & & \\
\hline Precirrchotic & 14 & 9 & 35 & & \\
\hline No fibrosis & 50 & & & 6 & 88 \\
\hline
\end{tabular}

\section{Discussion}

Percutaneous liver biopsy is required for the definitive assessment of parenchymal liver disease. Although both hepatic steatosis and fibrosis are diffuse processes, their distribution may vary, resulting in sampling error of up to $10 \% .{ }^{89}$ Despite this limitation liver histology was used as the gold standard in this study. Real time ultrasound scanning has the advantage over liver biopsy in providing a rapid overall assessment of liver morphology in addition to being routinely available and completely safe. For these reasons scanning fulfils two important criteria for a good screening procedure to detect liver disease. The major requirement for a screening procedure, however, is a high degree of sensitivity and specificity for detecting major liver diseases. In the practical management of treatable liver disease the detection of alcoholic liver 
disease should be emphasised, ideally before cirrhosis develops, allowing resources to be concentrated on this subgroup to prevent irreversible liver disease.

This study shows that ultrasound scanning performed during the course of the routine clinical workload was $87 \%$ sensitive and $89 \%$ specific in detecting fatty infiltration or fibrosis of the liver that had been proved by histology. The major disease missed was cirrhosis: three patients with cirrhosis had normal ultrasound scans; one had cryptogenic cirrhosis with metastatic liver disease (the metastatic disease was correctly identified); and two had primary biliary cirrhosis. In the largest subgroup, alcoholic liver disease, 47 patients $(94 \%)$ had abnormal ultrasound scans. Most (35) had potentially reversible precirrhotic disease.

When the diseases were considered separately scanning was more sensitive in detecting hepatic steatosis than fibrosis. All cases of moderate and severe fatty infiltration were identified, as were most mild cases. Steatosis due to a variety of aetiological factors was identified, including steatosis due to diabetes mellitus, methotrexate, amiodarone, and alcohol. Ultrasound scanning was highly specific in distinguishing fatty liver from normal liver (only one false positive result). Fibrosis was less reliably detected, with only $57 \%$ of patients with histologically increased fibrosis identified. The subgroup with established cirrhosis was more accurately identified: 15 patients $(71 \%)$ were shown to have increased fibrosis on scanning.

The lack of sensitivity in detecting fibrosis is only a theoretical limitation when testing for alcoholic liver disease, because of the frequent association of steatosis with fibrosis. Thus all patients with alcoholic liver disease in this series with increased fibrosis had abnormal liver echo patterns, although in many cases only the steatosis was specifically recognised because of the failure to detect pin head echoes within the fine echo pattern.

The wide variation in the reported sensitivity of ultrasound scanning in detecting hepatic steatosis from $9 \%{ }^{6}$ to $94 \%$ in this study is caused by two major factors. Firstly, there are important differences between our study and other series in the criteria for steatosis and fibrosis used, particularly the importance of posterior beam attenuation. Secondly, there is an important difference between the ultrasound transducers used. Two groups believe that minimal beam attenuation occurs in steatosis while increased beam attenuation signifies fibrosis. ${ }^{72}$ This view contrasts completely with our own. One reason for this discrepancy is that other workers have, in fact, observed the effect of severe steatosis associated with fibrosis. In vitro studies support our views; thus Bamber et al examined the acoustic properties of fat and collagen in the liver and found much higher attenuation with steatosis compared with fibrosis. ${ }^{18}$ We have also established our view in patients with histological evidence of fibrosis but no evidence of any appreciable steatosis. Interestingly, despite the use of different criteria other workers have arrived at detection rates remarkably similar to ours. ${ }^{7}$ This is probably because, although they made observations regarding beam penetration, their final assessment depended heavily on the characteristic fine echo pattern of steatosis and the coarse echo pattern of fibrosis.

The second major factor was the use of a $5 \mathrm{mHz}$ transducer in this study, in contrast to the $2.25 \mathrm{mHz}$ or $3.5 \mathrm{mHz}$ transducers used in other studies. Attenuation and backscatter of the sound beam is considerably less with a $3.5 \mathrm{mHz}$ than with a $5 \mathrm{mHz}$ beam, and thus the extent of posterior beam attenuation is more difficult to appreciate. The severity of steatosis is also important, as sensitivity increases with greater degrees of infiltration. Thus in this study the sensitivity in detecting moderate and severe steatosis was $100 \%$, while in an earlier retrospective study the sensitivity increased from $60 \%$ overall to $90 \%$ when only moderate and severe cases were considered." Unfortunately, the severity of steatosis was not assessed in the study of Meek et al. ${ }^{6}$

The specificity of scanning for liver disease is difficult to establish from our findings, as histological verification that our control group had normal livers was not considered to be justified. Nevertheless, three recent series that have compared histological and ultrasound examination of the liver in normal subjects all reached similar conclusions-namely, that false positive results were exceedingly rare, with specificity ranging from 94 to $100 \% .^{71112}$ In this study in the subgroup of patients with irritable bowel disease (who did not undergo biopsy but were presumed to have normal liver histology) there was one false positive result, resulting in a specificity of $99 \%$.

A recent report questioned the ability of ultrasound scanning to distinguish between steatosis and fibrosis. ${ }^{12}$ The authors suggested that in many cases fibrosis and steatosis occurred together and it was the fibrotic component that produced the increased echogenicity in "fatty" liver rather than the fat itself. The present series, however, shows that this theory is incorrect as 30 of 33 patients with pure steatosis and no increased fibrosis on liver biopsy produced abnormal echo patterns. The reason why scanning is less sensitive in detecting fibrosis is not clear. In mixtures of steatosis and fibrosis the features of steatosis often predominate, masking the fibrotic component. Less severe cases of fibrosis would be expected to be more difficult to distinguish because they produce minor echo abnormalities. Six cases of established cirrhosis were missed in this study. In four cases (two wrongly interpreted as showing moderate steatosis and two with severe steatosis) the error was almost certainly caused by difficulty in distinguishing between fatty infiltration and fibrosis existing together. Interestingly, the two other patients had primary biliary cirrhosis, and other authors have commented on the difficulty in detecting primary biliary cirrhosis by ultrasound scanning.?

It is accepted that alcoholic liver disease is underdiagnosed. Several screening tests have been used to increase detection, including standard biochemistry,${ }^{13}$ mean corpuscular volume,${ }^{14}$ and the presence of rib fractures on chest radiography. ${ }^{15}{ }^{16}$ Although individually, or particularly in combination, they may be accurate in detecting alcoholic liver disease, none provides any information regarding prognosis for the development of cirrhosis. ${ }^{16}$

The recognition of precirrhotic alcoholic liver disease may be more important than previously thought. Although hepatic steatosis is completely reversible with abstinence, recent work has shown that the degree of steatosis is a major prognostic factor for the development of cirrhosis. ${ }^{17}$ It is well known that only $15-20 \%$ of heavy alcohol abusers develop cirrhosis; thus there must be a factor that induces susceptibility. Steatosis seems to be a marker for this susceptibility. On the basis of our study routine ultrasound scanning can accurately identify patients with alcoholic liver disease who are at risk of developing cirrhosis at an early stage.

\section{References}

1 Wheeler PG, Theodossi A, Pickford R, Laws J, Knill-Jones RP, Williams R. Noninvasive techniques in the diagnosis of jaundice-ultrasound and computer. Gut 1979;20: 196-9. 2 Taylor KJW, Rosenfield AT. Grey scale ultrasonography in the differential diagnosis of obstructive jaundice. Arch Surg 1977;112:820-5.

3 Lawson TL. Hepatic abscess: ultrasound as an aid to diagnosis. Dig Dis Sci 1977;22:33-7.

4 Theodossi A, Spiegelhalter D, Portmann B, Eddelston AWF, Williams R. The value of clinical, biochemical, ultrasound and liver biopsy data in assessing patients with liver disease. Liver 1983;3:315-26.

5 Joseph AEA, Dewbury KC, McGuire PG. Alcohol in the detection of chronic liver disease. (The 'bright' liver.) Br f Radiol 1979;52:184-8.

6 Meek DR, Mills PR, Gray HW, Duncan JG, Russell RI, McKillop JH. A comparison of computer tomography, ultrasound and scintigraphy in the diagnosis of alcoholic liver disease. $\mathrm{Br} \mathcal{F}$ Radiol 1984;57:23-7.

7 Debognie JC, Pauls C, Fievez M, Wibin E. Prospective evaluation of the diagnostic accuracy of liver ultrasonography. Gut 1981;22:130-5.

8 Scott WW, Sanders RC, Siegelman SS. Irregular fatty infiltration of the liver-diagnostic dilemma. AfR 1980;134:67-71.

9 Czaja A, Steinberg AS, Saldana $M$, et al. Peritoneoscopy-its value in the diagnosis of liver disease. Gastrointest Endosc 1973;20:23-5.

10 Dewbury KC, Clark B. The accuracy of ultrasound in the detection of cirrhosis of the liver. $\mathrm{Br} \mathcal{J}$ Radiol 1979;52:945-8.

11 Foster KJ, Dewbury KC, Griffith AH, Wright R. The accuracy of ultrasound in the detection of fatty infiltration of the liver. Br $\mathcal{F}$ Radiol 1980;53:440-2.

12 Taylor KJ, Gorelick FS, Rosenfeld AT, Riely CA. Ultrasonography of alcoholic liver disease and histological correlation. Radiology 1981;141:157-61.

13 Levi AJ, Chalmers DM. Recognition of alcoholic liver disease in a district general hospital. Gu 1978;19:521-5.

14 Bernadt MW, Mumford J, Taylor C, et al. Comparison of questionnaire and laboratory tests in the detection of excessive drinking and alcoholism. Lancet 1982;i:325-31.

15 Lindsell DRM, Wilson AG, Maxwell JD. Fractures on the chest radiograph in detection of alcoholic liver disease. Br Med f 1982;285:597-9.

16 Maxwell JD, Patel SP, Bland JM, Lindsell DRM, Wilson AG. Chest radiography compared to laboratory markers in the detection of alcoholic liver disease. $7 R$ Coll Physicians Lond laboratory marker.

17 Sørensen TIA, Orholm M, Bentsen KD, Høbye G, Eghøje K, Christoffersen P. Prospective evaluation of alcohol abuse and alcoholic liver injury in men as predictors of development of cirrhosis. Lancet 1984;ii:241-4. 